\title{
Beerensortierung bei Weißwein nach Größe und Farbe und deren Einfluss auf die Weinqualität
}

\author{
Maximilian Freund ${ }^{1}$, Magali Lafontaine ${ }^{1}$, Kai-Uwe Vieth ${ }^{2}$, und Christian Negara ${ }^{2}$ \\ ${ }^{1}$ Hochschule Geisenheim University, Von-Lade-Strasse 1, 65366 Geisenheim, Germany \\ ${ }^{2}$ Fraunhofer - IOSB, Fraunhofer Strasse 1, 76131 Karlsruhe, Germany
}

\begin{abstract}
Im Zuge des verstärkten Maschineneinsatzes bei der Traubenlese kommt der optischen Traubenbzw. Beerensortierung für die Weinqualität eine zunehmende Bedeutung zu. Nicht nur das Aussortieren von Fremdkörpern und faulem Traubenmaterial, sondern auch die zusätzlichen Möglichkeiten der Selektion des von Natur aus heterogenen Beerenmaterials nach qualitätsbestimmenden Inhaltsstoffen stehen im Mittelpunkt. Da die optischen Daten unabhängig vom verwendeten Wellenlängenbereich vorwiegend über Reflektion gewonnen werden, bleiben die Informationen vorwiegend auf die Inhaltsstoffe der Beerenhaut beschränkt. Beereneigenschaften wie Größe und Farbe sind daher hilfreiche Parameter, von denen seit Längerem bekannt ist, dass sie einen Einfluss auf die Inhaltstoffe und somit auf die Weinstilistik nehmen. Die hier vorgestellten Untersuchungen zeigen zum einen, welche analytischen Eigenschaften sich durch Beerengröße und -farbe in der Beere selbst ändern, und zum anderen, welchen Einfluss diese Eigenschaften auf die daraus resultierende Weinqualität haben. Daraus wird abgeleitet, in wieweit eine optische Sortierung nach diesen beiden Parametern einen Einfluss auf den späteren Weinstil besitzt. Die Ergebnisse werden am Beispiel von Trauben der Rebsorte Vitis vinifera L. $c v$. Weißburgunder aus dem Weinanbaugebiet Rheingau, Deutschland, dargestellt.
\end{abstract}

\section{Einleitung}

\subsection{Optische Sortierung}

In den vergangenen Jahren hat die Sortierung von Trauben bzw. von Beeren immer mehr an Bedeutung gewonnen. Diese Entwicklung ist vor allem im Bereich der Produktion von hochwertigen Weinen und in der Weiterverarbeitung von Lesegut aus Maschinenlese zu beobachten.

Während die meisten Schritte der Traubenverarbeitung das Erhalten der Traubenqualität zum Ziel haben, besteht bei der Traubensortierung durch Aussonderung traubenfremder Bestandteile sowie grüner Pflanzenteile, unreifer, beschädigter oder angefaulter Beeren (MOG - material other than grapes) die Möglichkeit, die Qualität des Lesegutes aktiv zu erhöhen. Dies ist vor allem mit der Zunahme gerade der traubenfremden und traubeneigenen Verunreinigung, wie beispielsweise Stiele, Blätter, Insekten, Rebholz und Heftklammern, durch die Maschinenlese von Bedeutung.

Dies war in der Vergangenheit vorwiegend Handarbeit, sei es in Form der selektiven Handlese oder mittels manueller Sortiereinrichtungen wie z. B. Vibrationsoder Bandsortiertischen. Seit einiger Zeit gibt es auch automatische Sortieranlagen, die in Abhängigkeit ihrer Sortiertechnik nach unterschiedlichen Selektierkriterien sortieren.

Rein mechanische Sortiersysteme können mit Hilfe von Sieben, Rechen, Ausblasdüsen oder Dichtebädern vorwiegend traubenfremde sowie traubeneigene Verun- reinigungen entfernen und auch nach Beerengröße trennen. Einige dieser mechanischen Systeme sind mittlerweile ergänzend zum Entrapper auf Lesemaschinen integriert.

Die optischen Systeme erweitern die Möglichkeiten des Sortierens. So sind diese Anlagen in Abhängigkeit ihrer bildgebenden Technik in der Lage die möglichen Sortierkriterien über Größe, Form und Farbe hinaus auch auf qualitätsrelevante Inhaltsstoffe der Beeren $\mathrm{zu}$ erweitern.

So hat die Infrarot-Spektroskopie (IR) ergänzend zum sichtbaren Wellenlängenbereich mittlerweile eine weite Verbreitung in den unterschiedlichen Bereichen der Beeren-, Most- und Weinanalyse gewonnen [1,2]. Einen Überblick über die bei der optischen Beerensortierung derzeit geprüften Qualitätsparameter sind in Tabelle 1 zusammengetragen.

Da im Bereich der Beerensortierung die optischen Daten über Reflexion gewonnen werden, ist der eigentliche Informationsträger die Beerenhaut und die in ihr lokalisierten Inhaltsstoffe. Zusätzlich ist als Fehlerquelle der bei den vorbereiteten Schritten wie Lese, Transport, Entrappen frei werdende Saft, der sich auf der Beerenoberfläche befindet, zu beachten und gegebenenfalls in den Sortieralgorithmen der Software zu berücksichtigen.

Diese Überlegungen führen dazu, die Beerenfarbe und den von der Reflexion unabhängigen Parameter Beerengröße als Sortierkriterium einzubeziehen und deren Einfluss auf die Weinqualität zu betrachten. 
Tabelle 1. Zusammenhang zwischen analytischen und optischen Größen zur Charakterisierung der Beerenqualität.

\begin{tabular}{|l|c|c|}
\hline Parameter & Wellenlänge & Quelle \\
\hline Brix & $700-1200 \mathrm{~nm}$ & {$[3]$} \\
\hline Brix, pH-Wert, Anthocyane & $640-1100 \mathrm{~nm}$ & {$[4]$} \\
\hline Brix, Zucker, pH-Wert & $380-1650 \mathrm{~nm}$ & {$[5]$} \\
\hline Dichte, Anthocyane & $700 \mathrm{~nm}$ & {$[6]$} \\
\hline Flavonole, Gesamtphenole & $1100-2498 \mathrm{~nm}$ & {$[7]$} \\
\hline Dichte, Anthocyane & $400-1000 \mathrm{~nm}$ & {$[8]$} \\
\hline
\end{tabular}

\subsection{Beerengröße}

Vor allem bei der Rotweinbereitung wird die Beerengröße als wichtiger Parameter hinsichtlich der späteren Weinqualität angesehen. Als Grund wird das sich verschiebende Saft/Schalenverhältnis zu Gunsten der Beerenhaut und der in ihr lokalisierten Inhaltsstoffe - vor allem der Anthocyane - angeführt $[9,10]$. Zwar steigt mit zunehmender Größe der Beeren auch der Hautanteil und gleichzeitig die dort lokalisierten Inhaltstoffe proportional zur Beere [9], doch bei Bezug der Beerenhautinhaltsstoffe auf das Beerenfrischgewicht ergibt sich eine erhöhte Konzentration dieser Stoffe bei den kleineren Beeren [12].

Erklärt wird dieses Phänomen u. a. mit einer schnelleren Reife der kleinen Beeren, die zum einen mit den hormonellen Veränderungen der geringeren Kernzahl aber auch durch die höhere Verdunstung durch die größere relative Hautoberfläche begründet wird. Die dadurch bedingte stärkere Transpiration führt zu einer verstärkten Einlagerung von Inhaltsstoffen in die Beere [13]. In Abhängigkeit der Beerenexposition dürften sich kleinere Beeren zudem schneller erwärmen und somit die Transpiration, aber auch die Stoffwechselprozesse u. a. auch den Äpfelsäureabbau - beschleunigen.

Aufbauend auf diesen Überlegungen scheinen hinsichtlich des Sortierkriteriums Beerengröße - unabhängig ob weiße oder rote Beeren - vor allem die Inhaltsstoffe von Bedeutung, die in der Beerenhaut lokalisiert sind. Dies trifft vor allem für die in der Weißweinbereitung bedeutenden Aromakomponenten und Mineralstoffe, wie Kalium, zu [12]. Die Abhängigkeit der Säure von der Beerengröße ist dagegen differenziert zu betrachten. Weinsäure liegt in höherer Konzentration in der Beerenhaut vor, Apfelsäure ist gleichmäßig über die Beere verteilt, wobei ihre relative Verteilung in der Beerenhaut im Vergleich zum Beerenfleisch während der Reife ansteigt [14-17]. Bei Weinen aus kleinen und großen Beeren der Rebsorte Vitis vinifera $L$. $c v$. Chenin Blanc aus Südafrika wurden keine Unterschiede bei den Wein-Estern gefunden, die Qualität der Weine aus kleineren Beeren wurde jedoch als besser bewertet [18].

\subsection{Beerenfarbe}

Mit zunehmender Reife verfärben sich die Beeren - rote von grün über rot nach dunkelblau, weiße von grün über gelb nach goldgelb. Bei den roten Beeren wird dies vorwiegend der Akkumulation von Anthocyanen zugesprochen, während bei weißen neben Polyphenolen wie Flavonoide (gelb-braun) auch Carotinoide (gelb-orange) für die Farbe verantwortlich gemacht werden. Das grüne Chlorophyll wird im Laufe der Reife sowohl bei roten als auch bei weißen Beeren abgebaut [19].

Da Carotinoide Aromavorstufen der $\mathrm{C}_{1} 3$-Norisoprenoide und der Terpene sind, gilt diese Stoffgruppe als wichtiges Qualitätskriterium. So konnte bei der Rebsorte Vitis vinifera L. $c v$. Riesling aus dem Rheingau, Deutschland eine Korrelation zwischen der Beerenfarbe gelb-rötlich (a* im CIELab-Farbkörperraum) und den glycosidisch gebundenen Aromavorstufen (GG) gezeigt werden [20]. Da diese mit dieser Methode mitbestimmten glycosidisch gebundenen Phenole nur einen geringen Zusammenhang mit der Beerenfarbe gelb-rötlich zeigen, können die GG-Werte als Indikator der Aromavorstufen angesehen werden.

Auch wird in verschiedenen Untersuchungen kein Zusammenhang zwischen Beerenfarbe und dem Zuckerhalt der Beeren festgestellt [20-22].

Für die Rebsorte Vitis vinifera L. $c v$. Sauvignon Blanc aus der Region Western Cape, Südafrika werden die in Tabelle 2 aufgelisteten Farbwerte entsprechenden Aromaprofilen zugeordnet. Auch bei den hier zugrunde liegenden Untersuchungen konnten kein direkter Zusammenhang zwischen der Beerenfarbe und dem Zuckergehalt bzw. der titrierbaren Gesamtsäure festgestellt werden [23].

Die im folgenden vorgestellten Untersuchungen verfolgen das Ziel aufzuzeigen, welchen Einfluss Beerengröße und -farbe bei der Rebsorte Vitis vinifera $L$. $c v$. Weißburgunder auf die Inhaltsstoffe der Beere nehmen und welche Möglichkeiten sich durch ein gezielten Ausbau nach den beiden Parametern Größe und Farbe für die Weinstilistik und die Weinqualität ergeben können.

\section{Material und methoden}

\subsection{Vorversuche}

In einem Vorversuch wurden jeweils 1000 Beeren nach Beerengröße und -farbe klassifiziert und untersucht. Davor wurden sie zuerst mit der Hand vom Stielgerüst befreit.

\subsubsection{Beerengröße}

Beerengrößensortierung: Die Beeren wurden an zwei Terminen (16.09.2013 und 06.10.2013) unter Verwendung von Sieben in sechs Größen $(6,3 \mathrm{~mm}, 8 \mathrm{~mm}, 10 \mathrm{~mm}$, $12,5 \mathrm{~mm}, 14 \mathrm{~mm}$ und $16 \mathrm{~mm}$ ) gruppiert. Die Beeren der beiden kleinsten Gruppen wurden verworfen, da ihr Anteil zum ersten Termin nur 8\% (16.09.2013) zum zweiten Termin nur 6\% (06.10.2013) der Gesamtstichprobe ausmachte. Die vier restlichen Gruppen wurden zu den Gruppen kleine $(10-12,5 \mathrm{~mm})$ und große Beeren (>12,5 mm) zusammengefasst.

Chemische Analysen: Das Einzelbeerengewicht wurde gewogen (METTLER AE200, Sartorius, Göttingen, Deutschland), bei einem Druck von 1 bar ausgepresst (Longarone 85, QS System GmbH, Norderstedt, Deutschland) und die gewonnen Moste gefiltert. Der Gesamtextrakt wurde als ${ }^{\circ}$ Oechsle mit einem Handrefraktometer (Leo Kübler GmbH, Karlsruhe, Deutschland) bestimmt. $1,5 \mathrm{~mL}$ des Saftes wurden fünf Minuten bei $1400 \mathrm{~g}$ zentrifugiert (MiniSpin ${ }^{\circledR}$ Plus, Eppendorf AG, Wesseling 
Tabelle 2. Zusammenhang zwischen analytischen und optischen Größen zur Charakterisierung der Beerenqualität [erweitertnach 23].

\begin{tabular}{|l|c|}
\hline Farbwertim HSL-Modell $\left[{ }^{\circ} \mathbf{F}\right]$ & Aromaprofil \\
\hline$>90$ & grün/unreif \\
\hline $90-85$ & grün/Spargel \\
\hline $85-80$ & Spargel/Citrus \\
\hline $80-75$ & tropische Frucht \\
\hline $75-70$ & gärig/Terpene \\
\hline $70-65$ & phenolisch/neutral/Terpene \\
\hline $65-60$ & \\
\hline
\end{tabular}

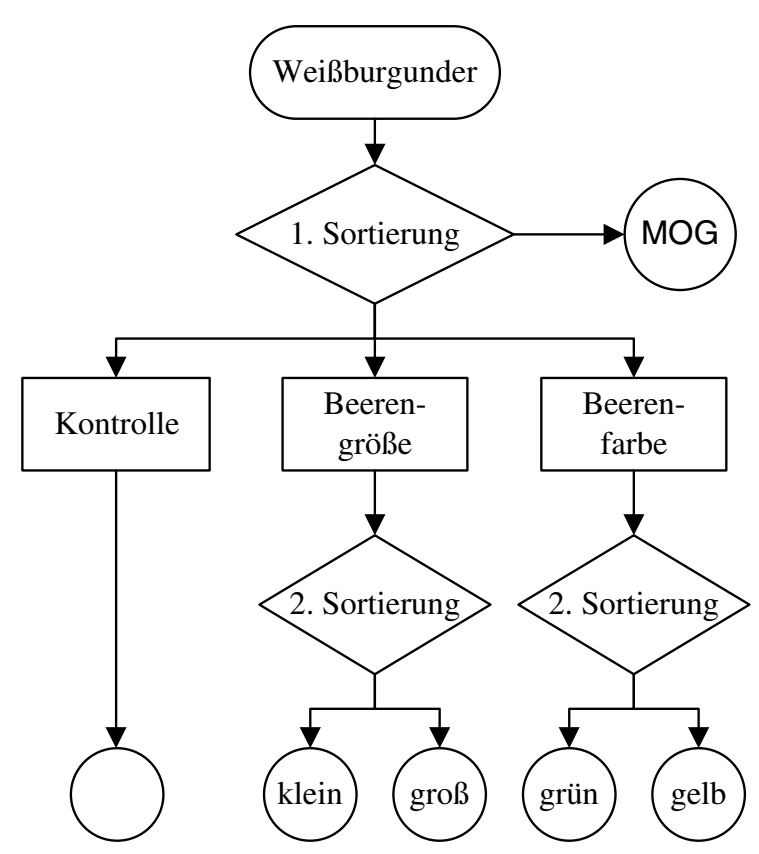

Abbildung 1. Versuchsaufbau Beerengröße und -farbe.

Berzdorf, Deutschland) und der Überstand mit Fouriertransform infrared spectroscopy (FTIR) (OenoFoss ${ }^{\mathrm{TM}}$, FOSS, Hillerød, Dänemark) für die Bestimmung der organischen Säuren, der titrierbaren Gesamtsäure, des $\mathrm{pH}$-Werts und des Gesamtextrakts herangezogen. Die Konzentration der primären Aminosäuren wurde im Most mit dem NOPA-Verfahren [24] als Isoleucin-Äquivalente bestimmt.

\subsubsection{Beerenfarbe}

Sortierung nach Beerenfarbe: Die Beeren wurden an drei Terminen (07.09.2012, 30.9.2012, 30.09.2013) mit Hilfe des Farbwertes a* im rot/grün-Bereich in 30 Gruppen aufgeteilt. Zur Farbwertbestimmung der Beeren wurde das Farbraummodell CIELab der Commission Internationale de l'Eclairage (CIE) verwendet. Hierzu wurden je $8 \mathrm{~mm}^{2}$ jeder Beere von zwei unterschiedlichen Seiten mit einem Spektrometer im sichtbaren Wellenlängenbereich (VIS) (Minolta 3500d, Konica Tokyo, Japan) aufgenommen und die Werte gemittelt. Die Parameter Helligkeit ( $\left.\mathrm{L}^{*}\right)$, rot/grün $\left(a^{*}\right)$ und gelb/blau $\left(b^{*}\right)$ wurden bei einem Standardbeobachter von $10^{\circ}$ und einer durchschnittlichen Tageslichtbeleuchtung (D65) bestimmt.
Glycosyl-Glucose-Analysen: Die nach der a*-Farbachse vorsortierten Beeren wurden mittels Glycosyl-GlucoseMethode [25] auf die glycosidisch gebundenen Aromavorstufen hinuntersucht.

Gesamtphenole: Die Gesamtphenole wurden nach der Folin-Ciocalteu-Methode untersucht und werden als Catechin-Äquivalente angeben.

\subsection{Großversuch}

Mit Hilfe der im Vorversuch ermittelten Informationen wurden die Trenngrenzen der Großversuche festgelegt.

Traubenherkunft: $1200 \mathrm{~kg}$ handgelesene Weißburgunder-Trauben mit einer Botrytisbefalls-haufigkeit von 57\% und eine Befallsstärke von 8\% (Lesedatum 09.10.2013) des Weingutes der Hochschule Geisenheim wurden in $25 \mathrm{~kg}$ fassende Einheitskisten gelesen und nach der Ernte in drei Partien Kontrolle $(193 \mathrm{~kg})$, Beerengröße $(531 \mathrm{~kg})$ und Beerenfarbe $(476 \mathrm{~kg})$ aufgeteilt.

Sortiervorgang: Für die Versuche wurde der Prototyp einer Beerensortieranlage des durch das Förderprogramm ZIM des BMWi geförderten Gemeinschaftsprojektes "GrapeSort" verwendet. Die Kisten wurden über einen Trichter per Hand mit Hilfe der Abbeermaschine Roto 10 (Armbruster, Güglingen-Frauenzimmer, Deutschland) abgebeert, von dort über ein Förderband mit Trichter (Armbruster) auf das Beschleunigungsband (Fraunhofer IOSB, Karlsruhe, Deutschland) der Sortiermaschine befördert, wo sie auf $3 \mathrm{~m} \mathrm{~s}^{-1}$ beschleunigt wurden. Mit Hilfe der optischen Sortiereinrichtung (Fraunhofer IOSB) und einem pneumatischen Düsenblock mit 128 Düsen (INWaG, Tübingen, Deutschland), der über das optische System gesteuert wird, wurde in Abhängigkeit der Fragestellung die beiden Fraktionen in $500 \mathrm{~kg}$ fassenden Kunststoffkisten getrennt aufgefangen.

Hierzu wurde in einem ersten Schritt die in den Vorversuchen gewonnen Klassifizierungsgrenzen für die Beerengröße bzw. -farbe in der Sortiersoftware hinterlegt. Vor der eigentlichen Sortierung erfolgte bei den drei Versuchen Kontrolle, Beerengröße und Beerenfarbe die Aussortierung der traubenfremden Bestandteile und der faulen Beeren, was in allen Varianten etwa $12 \%$ w/w ausmachte. Nach dieser ersten Sortierung wurde die Fraktion ohne traubenfremde Bestandteile in einem zweiten Durchgang in den Trichter des Förderbandes geschöpft und ein zweites Mal mit der Sortieranlage nach der Fragestellung Beerengröße bzw. -farbe sortiert. Während der Sortierung nach Größe bzw. Farbe wurde nach 
der Hälfte der verarbeiteten Menge die Sortierrichtung gewechselt, d. h. es wurden z. B. erst die großen Beeren und anschließend die kleinen Beeren mittels Druckluft aussortiert. Damit sollte einem möglichen Einfluss der Beschädigung der ausgeschossenen Beeren durch die Luftdüsen entgegnet werden.

Die Trauben wurden vor und die einzelnen Sortierfraktionen (Rappen, traubenfremde Bestandteile, anfallender Saft und die jeweiligen Beerenfraktionen) nach den Sortiervorgängen gewogen. Die Trauben der Kontrolle wurden nur entrappt und einmal über die Sortieranlage geführt, um die traubenfremden Bestandteile und die faulen Beeren zu entfernen.

Beerenbonitur: Jede fünf Minuten wurden $\mathrm{rd} .10 \mathrm{~kg}$ jeder Fraktion entnommen. Nach Versuchsende wurde aus den Einzelproben eine Gesamtprobe zu $10 \mathrm{~kg}$ zusammengestellt, die in Abhängigkeit der Fragestellung auf die Parameter traubenfremde Bestandteile, Beerengröße und farbe bonitiert wurden.

Mostbehandlung: Nach dem Wiegen gelangten die Beerenfraktionen getrennt mit Hilfe eines Gabelstaplers mit Drehkranz in die pneumatische Presse Europress EHP 300 (Scharfenberger, Bad Dürkheim, Deutschland), wo sie mit Hilfe des Standardprogramms 3 (max. 2 bar, $118 \mathrm{~min}$ ) abgepresst wurden. Dem Abpressen folgte eine Schwefelung mit $35 \mathrm{mg} \mathrm{L}^{-1}$ einer 5\%igen Lösung und eine Vorklärung mittels Sedimentation über $18 \mathrm{~h}$.

Gärung und Weinbereitung: Die Gärung erfolgte je Variante in je einer $50 \mathrm{~L}$ und $25 \mathrm{~L}$ großen Korbflasche, die zu $95 \%$ befüllt wurden. $200 \mathrm{mg} \mathrm{L}^{-1}$ Hefe (Oenoferm Klosterneuburg, Erbslöh, Geisenheim, Deutschland) wurde nach Rehydration gemäß Herstellerangaben zugegeben. Es wurde der Gärverlauf durch tägliches Bestimmen der Dichte und der Temperatur (DMA 35n, Anton Paar, Graz, Österreich) kontrolliert. Nach der Gärung erfolgte ein Beifüllen der Korbflaschen. Vier Wochen nach dem Beifüllen wurden die Weine von der Hefe abgetrennt und mit $100 \mathrm{mg} \mathrm{L}^{-1}$ einer 5\%igen Lösung geschwefelt. Acht Wochen nach dem Abstich erfolgte, wenn notwendig, eine Einstellung der freien schwefligen Säure auf $45 \mathrm{mg} \mathrm{L}^{-1}$ und die Abfüllung über eine Sterilfiltration (Seitz $\mathrm{EK}^{\circledR}$, Pall, Bad Kreuznach, Deutschland) und einen dreistelligen Gleichdruckfüller im Normaldruckbereich TA3 (KVTTroxler, Ettenheim, Deutschland).

Chemische Analysen: Die nach der Vorklärung genommenen Proben wurden gefiltert und untersucht. Die Weine wurden nach der Abfüllung bezüglich Alkohol, vergärbaren Zucker, Gesamtextrakt, zuckerfreien Extrakt, titrierbare Gesamtsäure, Weinsäure und $\mathrm{pH}$-Wert mittels FTIR (WineScan ${ }^{\mathrm{TM}}$, FOSS, Hillerød, Dänemark) analysiert. Die freie und gesamte schweflige Säure wurden mittels jodometrischer Titration bestimmt.

Sensorik: Alle Weine wurden in einem Weinkeller bei $15^{\circ} \mathrm{C}$ für zwei Monate gelagert. Die Sensorik fand im Sensorikraum des Instituts für Oenologie der Hochschule Geisenheim mit dem hochschuleigenen Prüferpanel statt. Als Methoden kamen Dreieckstest- und Rangordnungsprüfung zum Einsatz. Die Ergebnisse wurden mit der FIZZ-Software (Version 2.47B, Biosystèmes, Couternon, Frankreich) ausgewertet.

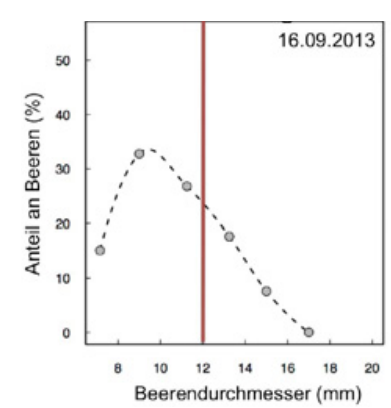

(a)

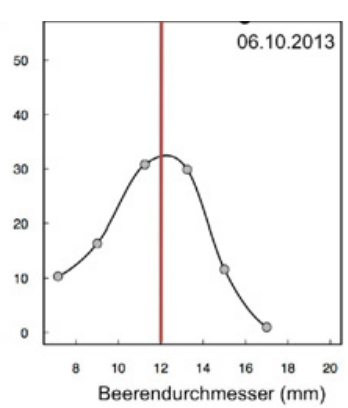

(b)
Abbildung 2. relative Häufigkeit des Beerendurchmessers der Weißburgunder-Beeren zu den Terminen (a) 16.09.2013 und (b) 06.10.2013.

\section{Ergebnis}

\subsection{Beerengröße}

\subsubsection{Vorversuch}

Abbildung 2 zeigt die Größenverteilung der WeißburgunderBeeren zu den beiden Terminen 16.9.2013 und 06.10.2013.

Es wird deutlich, dass sich mit zunehmender Reife der Beerendurchmesser vergrößert. Zur Festlegung der Trenngrenze für den Großversuch, wurde der Median bzw. die 50\% Quantile zum lesenahen Termin am 06.10.2013 benötigt, die bei $12 \mathrm{~mm}$ liegt, so dass die Größenklassen $<10$ und $10-12,5 \mathrm{~mm}$ zu der Variante kleine Beeren und die Größenklassen >12,5-14 mm, 14-16 mm und >16 mm zu der Variante große Beeren vereinigt wurden.

In Tabelle 3 sind die Ergebnisse der Beeren- und der Mostanalyse des Vorversuchs zusammengefasst. Zum ersten Zeitpunkt ist $\mathrm{zu}$ erkennen, dass der Most aus kleineren Beeren im Durchschnitt ein höheres Mostgewicht aufweist. Zum zweiten Zeitpunkt wird dies nicht bestätigt. Hingegen zeigt sich zum zweiten Zeitpunkt, dass der Gehalt an Äpfelsäure und hefeverfügbarem Stickstoff bei den kleineren Beeren niedriger als bei den größeren Beeren war. Der Weinsäuregehalt ist dagegen bei den kleineren Beeren mit $0,72 \mathrm{~g} \mathrm{~L}^{-1}$ deutlich höher. Diese Erkenntnisse decken sich mit weiteren Untersuchungen bei der Rebsorte Riesling, die innerhalb dieses Projekts durchgeführt wurden.

Hinsichtlich der Anzahl der Beeren liegt die Trenngrenze von 12,5 mm mit $47 \mathrm{zu} 53 \%$ mittig. Das mittlere Beerengewicht der kleinen Beeren zum Zeitpunkt 06.10.2013 liegt mit $0,9 \mathrm{~g} \mathrm{zu} 1,70 \mathrm{~g}$ um $47 \%$ niedriger als bei den großen, somit ist das Gesamtgewicht der kleinen Beeren auch entsprechend niedriger, weshalb bei den Großversuchen mit einer Trenngrenze von $13 \mathrm{~mm}$ gearbeitet wurde.

\subsubsection{Großversuch}

Bei Betrachtung der Massenbilanz (vgl. Abbildung 3) zeigt sich, dass die Aufgabe zwei gleichgroße Partien zu sortieren mit 32 zu 33\% w/w erfüllt wird. Die Genauigkeit der Sortierung liegt bei den kleinen Beeren bei 80 und bei den großen bei $89 \%$. 
Tabelle 3. Beeren- und Mostanalysen der Varianten kleine Beeren und große Beeren der Rebsorte Weißburgunder zu den Terminen 16.09.2013 und 06.10.2013 des Vorversuchs Beerengröße.

\begin{tabular}{|c|c|c|c|c|}
\hline \multirow{2}{*}{ Parameter } & \multicolumn{2}{|c|}{16.09 .2013} & \multicolumn{2}{|c|}{06.10 .2013} \\
\hline & $\begin{array}{l}\text { kleine } \\
\text { Beeren }\end{array}$ & $\begin{array}{l}\text { große } \\
\text { Beeren }\end{array}$ & $\begin{array}{l}\text { kleine } \\
\text { Beeren }\end{array}$ & $\begin{array}{l}\text { große } \\
\text { Beeren }\end{array}$ \\
\hline Gesamtanzahl [\%] & 70 & 30 & 53 & 47 \\
\hline Gesamtgewicht [\%] & 54 & 46 & 37 & 63 \\
\hline Beerengewicht [g] & 0,87 & 1,35 & 0,90 & 1,70 \\
\hline Mostgewicht $\left[{ }^{\circ} \mathrm{Oe}\right]$ & 83,4 & 78,8 & 100,6 & 101,4 \\
\hline Gesamtsäure $\left[\mathrm{g} \mathrm{L}^{-1}\right]$ & 9,33 & 9,41 & 7,33 & 7,31 \\
\hline Weinsäure $\left[\mathrm{g} \mathrm{L}^{-1}\right]$ & 4,93 & 4,95 & 4,30 & 3,58 \\
\hline Äpfelsäure $\left[\mathrm{g} \mathrm{L}^{-1}\right.$ ] & 4,40 & 4,46 & 3,03 & 3,73 \\
\hline pH-Wert [] & 3,11 & 3,09 & 3,11 & 3,13 \\
\hline NOPA $\left[\mathrm{mg} \mathrm{L}^{-1}\right]$ & 95 & 127 & 178 & 210 \\
\hline
\end{tabular}

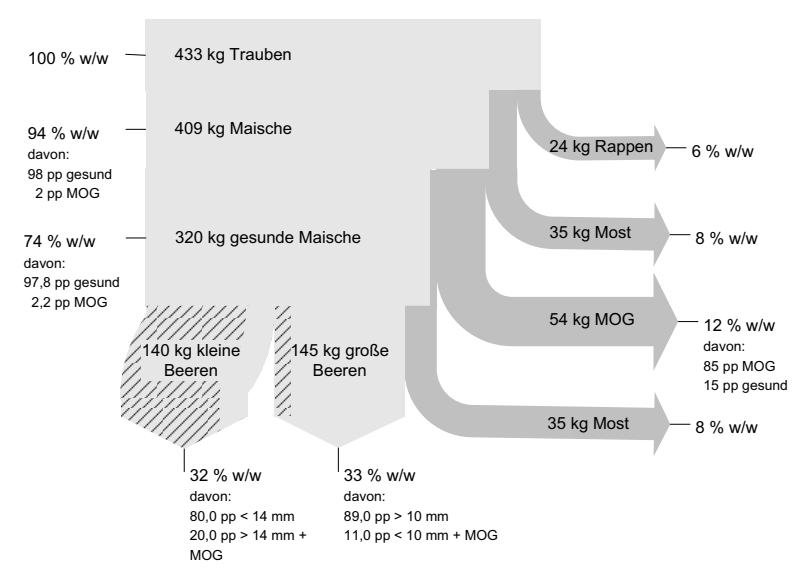

Abbildung 3. Massenbilanz der Sortierung nach Beerengröße Varianten kleine Beeren und große Beeren der Rebsorte Weißburgunder zum Lesetermin am 09.10.2013.

Tabelle 4. Mostanalysen der Varianten kleine Beeren und große Beeren der Rebsorte Weißburgunder zum Lesetermin am 09.10.2013.

\begin{tabular}{|c|c|c|}
\hline Parameter & kleine Beeren & große Beeren \\
\hline Gesamtgewicht [kg] & 140 & 145 \\
\hline Mostgewicht [ $\left.{ }^{\circ} \mathrm{Oe}\right]$ & 94 & 94 \\
\hline Gesamtsäure $\left[\mathrm{g} \mathrm{L}^{-1}\right]$ & 9,10 & 9,50 \\
\hline Weinsäure $\left[\mathrm{g} \mathrm{L}^{-1}\right]$ & 5,90 & 5,70 \\
\hline Äpfelsäure [g L ${ }^{-1}$ ] & 3,70 & 4,10 \\
\hline pH-Wert [] & 3,27 & 3,23 \\
\hline NOPA $\left[\mathrm{mg} \mathrm{L}^{-1}\right]$ & 212 & 235 \\
\hline
\end{tabular}

Diese Abweichungen erklären sich aus dem Bestreben zwei gleichgroße Partien zu erzeugen, so dass während der Sortierung die Größenverteilung angepasst werden musste. Auffällig ist der Gesamtverlust von 16\% w/w an Most, der sich aber aufgrund des Prototyps und der Arbeitsweise erklären lässt.

Die Analysen der vorgeklärten Moste sind in Tabelle 4 zusammengefasst. Die Unterschiede sind aufgrund der angestrebten Aufteilung in zwei gleichgroße Partien weniger deutlich als in den Vorversuchen (vgl. Tabelle 3). So ist das Mostgewicht beider Varianten identisch. Bei der titrierbaren Gesamtsäure weisen die großen Beeren

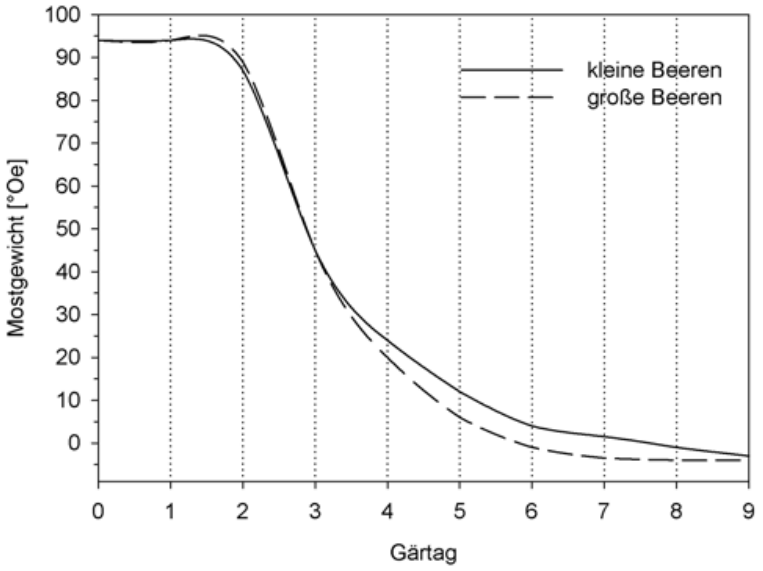

Abbildung 4. Gärverlauf der Varianten kleine Beeren und große Beeren der Rebsorte Weißburgunder im Jahr 2013.

mit 9,50 $\mathrm{g} \mathrm{L}^{-1} 0,4 \mathrm{~g} \mathrm{~L}^{-1}$ mehr Säure auf als die kleinen, was über den höheren Äpfelsäuregehalt der größeren Beeren zu erklären ist. Der Weinsäuregehalt ist wie bei den Vorversuchen bei den kleinen Beeren höher, fällt mit $0,2 \mathrm{~g} \mathrm{~L}^{-1}$ jedoch geringer aus. Der $\mathrm{pH}$-Wert beider Varianten ist vergleichbar, dagegen der hefeverfügbare Stickstoff mit $235 \mathrm{mg} \mathrm{L}^{-1} \mathrm{zu} 212 \mathrm{mg} \mathrm{L}^{-1}$ bei den großen Beeren höher.

Es kann nur spekuliert werden, in wie weit sich dieser höhere hefeverfügbare Stickstoff auf die Gärung auswirkt. Es zeigt sich jedoch, dass die Variante große Beeren im Gärverlauf (vgl. Abbildung 4) ab dem vierten Gärtag einer schnelleren Mostgewichtsabnahme unterliegt.

Auch der verbleibende geringere Rest an vergärbarem Zucker von $0,4 \mathrm{zu} 1,6 \mathrm{~g} \mathrm{~L}^{-1}$ belegt die vollständigere Gärung. Im Gegensatz zum Most sind die Gehalte an titrierbarer Gesamtsäure beider Varianten mit $8,65 \mathrm{~g} \mathrm{~L}^{-1}$ identisch (vgl. Tabelle 5).

Da keine malolaktische Gärung stattgefunden hat, lässt sich diese Säureminderung im Vergleich zum Most über den Weinsteinausfall und die damit verbundene Weinsäureverminderung erklären, die bei beiden Varianten im Vergleich zum Moststadium um $1 \mathrm{~g} \mathrm{~L}^{-1}$ beträgt. Somit weisen die Weine aus kleinen Beeren einen größeren Weinsäure- und einen kleineren Äpfelsäureanteil auf als die Weine aus großen Beeren. 
Tabelle 5. Weinanalysen der Varianten kleine Beeren und große Beeren der Rebsorte Weißburgunder im Jahr 2013.

\begin{tabular}{|c|c|c|}
\hline Parameter & kleine Beeren & große Beeren \\
\hline vorhandener Alkohol [\% v/v] & 13,1 & 13,2 \\
\hline vergärbarer Zucker [g L $\left.{ }^{-1}\right]$ & 1,6 & 0,4 \\
\hline Gesamtsäure $\left[\mathrm{g} \mathrm{L}^{-1}\right]$ & 8,65 & 8,65 \\
\hline Weinsäure $\left[\mathrm{g} \mathrm{L}^{-1}\right]$ & 4,90 & 3,90 \\
\hline pH-Wert [] & 3,25 & 3,25 \\
\hline zuckerfreier Extrakt $\left[\mathrm{g} \mathrm{L}^{-1}\right.$ ] & 23,1 & 22,8 \\
\hline Gesamtphenole [mg L $\left.{ }^{-1}\right]$ & 269 & 260 \\
\hline
\end{tabular}

Der um $0,3 \mathrm{~g} \mathrm{~L} \mathrm{~L}^{-1}$ höhere zuckerfreie Extrakt des Weines aus kleinen Beeren ist als gering einzustufen und eventuell über den höheren Anteil der in der Beerenhaut lokalisierten Mineralstoffe, wie Kalium, zu erklären. Auch der Gesamtphenolgehalt ist mit $9 \mathrm{mg} \mathrm{L}^{-1}$ als vergleichbar anzusehen.

Die vergleichbaren analytischen Werte spiegeln sich auch in der Sensorik wider. Beim durchgeführten Dreieckstest haben 8 von 14 Prüfern $(P=0,31 \geq 0,05)$ die Varianten kleine und große Beeren differenzieren können.

Somit muss aufgrund der Ergebnisse davon ausgegangen werden, dass es zwischen den Weinen aus kleinen und großen Beeren im Falle der vorliegenden Untersuchung bei einem 2013er Weißburgunder aus dem Rheingau/Deutschland keine analytischen und sensorischen Unterschiede gibt. Die Ergebnisse der Vorversuche zeigen jedoch, dass es unter Einhaltung einer klaren Trenngrenze möglich ist, Moste bzw. Weine unterschiedlicher Zusammensetzung aus kleinen und großen Beeren zu gewinnen und so den Weinstil zu beeinflussen. Weiter wird verdeutlicht, dass mit einer optischen Sortieranlage eine ausreichend genaue Trennung von Beeren unterschiedlicher Größe möglich ist.

\subsection{Beerenfarbe}

\subsubsection{Vorversuch}

Abbildung 5 zeigt die Farbverteilung des a*-Farbwerts des CIELab-System der Weißburgunder-Beeren zu den beiden Terminen 07.9.2012 und 30.09.2012. Es wird deutlich, dass die Farbe der Beeren von grün (negative $\mathrm{a}^{*}$-Wert) nach gelb-rot (positive $\mathrm{a}^{*}$-Wert) sich mit der Reife verschiebt. Beim Vergleich der beiden Termine wird gleichzeitig ersichtlich, dass mit zunehmender Reife auch die der Farbvariabilität zunimmt.

Die nach $\mathrm{a}^{*}$-Wert sortierten Beeren wurden auf Mostgewicht, glycosidisch gebundene Glucose (GGWerte) und Gesamtphenole analysiert.

Hinsichtlich des Mostgewichts konnte kein Zusammenhang festgestellt werden. Dagegen zeigt Abbildung 6, dass ein Anstieg der GG-Werte mit zunehmender Verfärbung zu beobachten ist. Da die Gesamtphenole nicht mit den GG-Werten ansteigen, kann angenommen werden dass durch eine Sortierung von Weißburgunder-Beeren nach Farbe im grün-rot Bereich sich die Aromaausprägung der aus ihnen gewonnenen Weine variieren lässt.

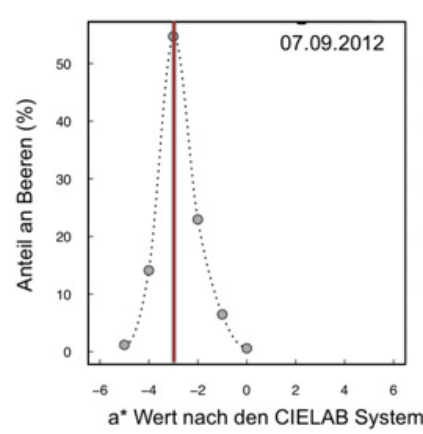

(a)

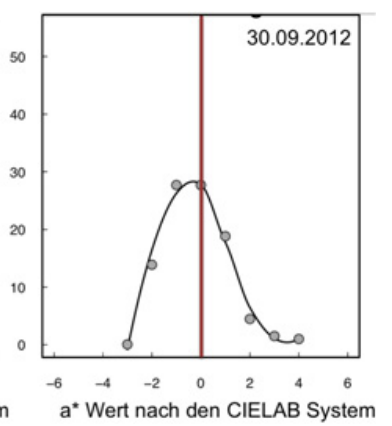

(b)
Abbildung 5. Relative Häufigkeit der Beerenfarbe nach a*-Wert nach der CIELab System der Weißburgunder-Beeren an den Terminen (a) 07.09.2012 (b) 30.09.2012.

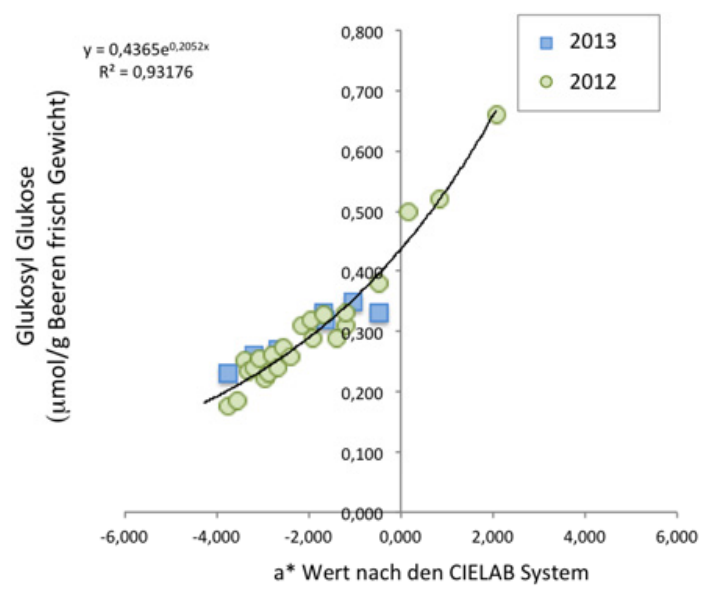

Abbildung 6. Korrelation des $a^{*}$-Farbwert des CIELabSystems mit glycosidisch gebundenen Aromavorstufen (GG als $\mu \mathrm{mol} \mathrm{g}^{-1}$ Beerenfrischgewicht) für Beerenhomogenisat aus Weißburgunder-Beeren für die Jahre 2012 und 2013.

\subsubsection{Großversuch}

Das Sortierergebnis der in Abbildung 7 zusammengefassten Massenbilanz der Beerenfarbe bestätigt mit einer Genauigkeit von $99 \%$ bei den gelben und $96 \%$ bei den grünen Beeren die hervorragende Selektionsleistung der Sortieranlage. Mit je 33\% w/w Anteil von grünen und gelben Beeren wurde auch die Aufgabe gleich große Partien zu erzeugen, erfüllt. Wie bereits bei dem Versuch Beerengröße fällt der hohe Gesamtmostverlust von $16 \% \mathrm{w} / \mathrm{w}$ auf, der zum einen auf die zwei Durchgänge durch die Sortieranlage und zum anderen auf die Pilotanlage selbst und deren mittlerweile behobenen konstruktiven Mängel zurückzuführen ist.

Bei der in Tabelle 6 dargestellten Mostanalyse fallen vor allem die Gesamtsäurewerte auf. Hier weisen die gelben Beeren mit 9,21 $\mathrm{g} \mathrm{L}^{-1}$ einen um $0,38 \mathrm{~g} \mathrm{~L}^{-1}$ höheren Gehalt auf als die grünen Beeren.

Die Einzelsäuren zeigen ein differenziertes Bild. Die Moste der Variante grünen Beeren haben mit 4,90 $\mathrm{g} \mathrm{L}^{-1}$ einen niedrigen Gehalt an Weinsäure, dagegen weisen sei eine um $0,72 \mathrm{~g} \mathrm{~L}^{-1}$ höhere Äpfelsäurekonzentration auf. Mostgewicht, pH-Wert und NOPA-Gehalt sind für beide Varianten als gleich anzusehen. 


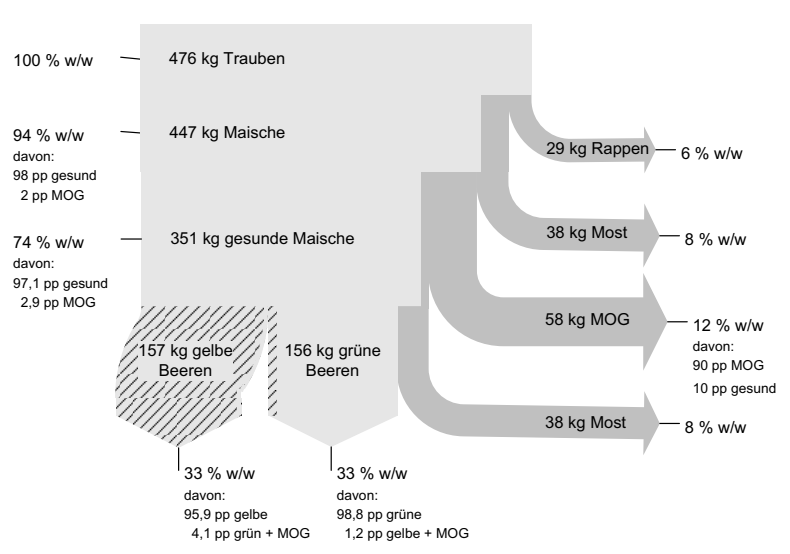

Abbildung 7. Massenbilanz der Sortierung nach Beerenfarbe der Varianten grüne Beeren und gelbe Beeren der Rebsorte Weißburgunder zum Lesetermin am 09.10.2013.

Tabelle 6. Mostanalysen der Varianten grüne Beeren und gelbe Beeren der Rebsorte Weißburgunder zum Lesetermin am 09.10.2013.

\begin{tabular}{|l|l|l|}
\hline Parameter & grüne Beeren & gelbe Beeren \\
\hline Gesamtgewicht $[\mathrm{kg}]$ & 156 & 157 \\
\hline Mostgewicht $\left[{ }^{\circ} \mathrm{Oe}\right]$ & 93 & 94 \\
\hline Gesamtsäure $\left[\mathrm{g} \mathrm{L}^{-1}\right]$ & 9,83 & 9,21 \\
\hline Weinsäure $\left[\mathrm{g} \mathrm{L}^{-1}\right]$ & 4,90 & 5,20 \\
\hline Äpfelsäure $\left[\mathrm{g} \mathrm{L}^{-1}\right]$ & 4,80 & 4,08 \\
\hline pH-Wert [] & 3,31 & 3,32 \\
\hline NOPA $\left[\mathrm{mg} \mathrm{L}^{-1}\right]$ & 213 & 212 \\
\hline
\end{tabular}

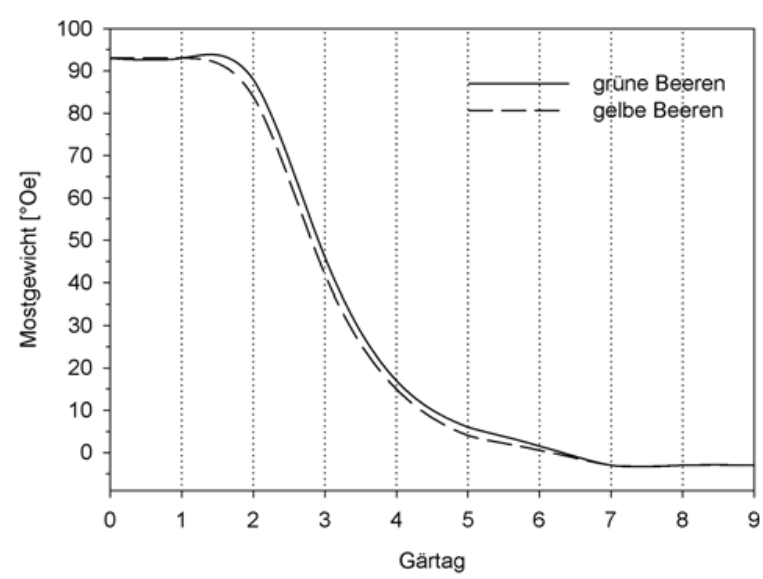

Abbildung 8. Gärverlauf der Varianten grüne Beeren und gelbe Beeren der Rebsorte Weißburgunder im Jahr 2013.

Diese vergleichbaren Mostwerte spiegeln sich auch in einem vergleichbaren Gärverlauf (vgl. Abbildung 8), dem vorhandenen Alkohol und dem vergärbaren Zuckerrest wider (vgl. Tabelle 7).

Mit $8,4 \mathrm{~g} \mathrm{~L}^{-1} \mathrm{zu} 8,5 \mathrm{~g} \mathrm{~L}^{-1}$ hat sich die titrierbare Gesamtsäure zwischen den grünen und gelben Beeren im Vergleich zu den Mostwerten angepasst. Diese Anpassung ist über den Weinsteinausfall und die dadurch zu beobachtende Veränderung der Weinsäure zu erklären. Lediglich die Gesamtphenole der Weine aus gelben Beeren weisen mit $289 \mathrm{mg} \mathrm{L}^{-1}$ einen um $15 \mathrm{mg} \mathrm{L}^{-1}$ höheren Wert als die Weine aus grünen Beeren auf. Diese Unterschiede
Tabelle 7. Weinanalysen der Varianten grüne Beeren und gelbe Beeren der Rebsorte Weißburgunder im Jahr 2013.

\begin{tabular}{|l|l|l|}
\hline Parameter & grüne Beeren & gelbe Beeren \\
\hline vorhandener Alkohol [\% v/v] & 13,1 & 13,1 \\
\hline vergärbarer Zucker $\left[\mathrm{g} \mathrm{L}^{-1}\right]$ & 0,3 & 0,7 \\
\hline Gesamtsäure $\left[\mathrm{g} \mathrm{L}^{-1}\right]$ & 8,4 & 8,5 \\
\hline Weinsäure $\left[\mathrm{g} \mathrm{L}^{-1}\right]$ & 3,9 & 4,3 \\
\hline pH-Wert [] & 3,30 & 3,30 \\
\hline zuckerfreier Extrakt $\left[\mathrm{g} \mathrm{L}^{-1}\right]$ & 23,3 & 23,6 \\
\hline Gesamtphenole $\left[\mathrm{mg} \mathrm{L}^{-1}\right]$ & 274 & 289 \\
\hline
\end{tabular}

haben sensorisch keine Auswirkungen, was die Ergebnisse des Dreieckstest belegen. Die 4 richtigen Antworten bei 14 Prüfern ( $\mathrm{P}=0,74 \geq 0,05)$ verdeutlichen, dass die Prüfer die Weine aus den Varianten grüne und gelbe Beeren nicht unterscheiden können.

Aufgrund der dargestellten Ergebnisse kann zwischen Weinen aus grünen und gelben Beeren im Falle der vorliegenden Untersuchung bei einem 2013er Weißburgunder aus dem Rheingau/Deutschland keine analytischen und sensorischen Unterschiede konstatiert werden.

\section{Fazit}

Die in der vorgestellten Untersuchung verfolgte Fragestellung, welchen Einfluss Beerengröße und -farbe bei der Rebsorte Vitis vinifera L. $c v$. Weißburgunder auf die Inhaltsstoffe der Beere nehmen und welche Möglichkeiten sich durch einen gezielten Weinausbau nach den beiden Parametern Größe und Farbe für die Weinstilistik und die Weinqualität ergeben können, kann bedingt beantwortet werden.

So bestätigen die Vorversuche des Parameters Beerengröße den Einfluss auf die Konzentration bestimmter Beereninhaltsstoffe. Es ist eine Differenzierung bei der Wein- und Äpfelsäure zu erkennen. Die Moste, die aus den kleineren Beeren gewonnen wurden, weisen eine deutlich geringere Äpfelsäure auf, was über eine schnellere Erwärmung dadurch hervorgerufene stärkere Dissimilation erklärt werden kann. Auch der höhere Weinsäuregehalt kann über die vermutete beschleunigte Reife der kleineren Beeren erklärt werden. Dagegen sprechen der Zucker in Form des Mostgewichts und der höhere NOPA-Gehalt der Moste aus großen Beeren gegen die Vermutung eines Reifevorsprungs kleinerer Beeren. Da beide Inhaltstoffe vorwiegend im Bereich des Fruchtfleisches vorkommen, ist jedoch eine Überlagerung des Reifeeffektes durch den aufgrund der Beerengröße höheren Fruchtfleischanteil zu erklären.

Die im Rahmen des Großversuchs zur Beerengröße durchgeführten Mostanalysen bestätigen die Ergebnisse der Vorversuche in den Aussagen des Mostgewichts, der Äpfelsäure und des Gehaltes an hefeverfügbarem Stickstoff. Bei der Weinsäure lassen sich auch größere Konzentrationen bei den Mosten aus kleinen Beeren nachweisen, doch nicht in der Höhe wie im Vorversuch. Auch die Weinanalysen verhalten sich konform. Der zusätzlich bestimmte Gesamtphenolgehalt weist zwar höhere Werte bei den Weinen aus kleineren Beeren auf, 
doch fallen diese mit $9 \mathrm{mg} \mathrm{L}^{-1}$ vernachlässigbar gering aus, so dass sich auch in der Sensorik keine absicherbaren Unterschiede feststellen lassen.

Auch bei dem Vorversuch der Beerenfarbe zeigt sich vor allem bei dem hier dargestellten Verlauf der glycosidisch gebundenen Aromavorstufen ein starker Zusammenhang mit der gelb-rötlichen Beerenfarbe. Die Mostanalysen des Großversuches zeigen jedoch, dass diese reifeabhängige Bildung der Aromavorstufen nicht gleichbedeutend mit der Erhöhung weiterer Reifeindikatoren wie Mostgewicht und hefeverfügbarem Stickstoff ist, da die Moste aus grünen und gelben Beeren vergleichbare Mostgewichte und NOPA-Gehalte aufweisen. Die titrierbare Gesamtsäure zeigt dagegen den erwarteten Verlauf. Sie ist bei den grünen Beeren höher. Wobei sich die Hauptsäuren unterschiedlich verhalten. Die Äpfelsäure ist bei Mosten aus gelben Beeren stärker vermindert. Hierfür wird die für die Verfärbung verantwortliche intensivere Strahlenexposition dieser Beeren und die dadurch erhöhte Erwärmung der Beeren und die beschleunigte Veratmung verantwortlich gemacht. Die Weinsäure ist dagegen bei den Mosten aus gelben Beeren etwas höher. Nach der Gärung kommt es zum Angleichen beider Varianten, so dass auch bei der Beerenfarbe zwischen den Weinen aus grünen und gelben Beeren kein signifikanter Unterschied festgestellt werden kann. Auch der um $15 \mathrm{mg} \mathrm{L}^{-1}$ höhere Gesamtphenolgehalt der Weine aus gelben Beeren beeinflusst das Ergebnis nicht.

Die in der Literatur gefundenen Erkenntnisse und die bei den vorgestellten Vorversuchen gewonnenen Ergebnisse zeigen, dass Beerengröße sowie -farbe geeignet sind, um den Weinstil auch bei Weißweintrauben über eine Sortierung nach diesen Kriterien $\mathrm{zu}$ beeinflussen. Auch haben die Versuche gezeigt, dass die Sortiertechnik in der Lage ist, diese Aufgabe mit für die Praxis mehr als ausreichender Zufriedenheit zu erfüllen. Dass dieses Ziel bei den hier vorgestellten Großversuchen nicht belegt werden konnte, lässt sich über die bewusst angestrebten zwei gleichgroßen Partien erklären. Bei stärkerer Ausnutzung der in den Vorversuchen beobachteten Variabilität sowohl der Beerengröße als auch der -farbe, lassen sich, wie nicht dargestellte Untersuchungen belegen, größere Unterschiede in den genannten Analyseparametern erzeugen, die sich dann vermutlich auch auf den Weinstil auswirken werden. In weiteren Versuchen soll deshalb mit größeren Traubenmengen gearbeitet werden, die dann einen praxisrelevanten Weinausbau ermöglichen.

\section{Literaturhinweise}

[1] R. Dambergs, M. Gishen, D. Cozzolino, Applied Spectroscopy Reviews 50, 17 (2015)

[2] M. Urbano-Cuadradoa, M.D. Luque de Castroa, P.M. Pérez-Juan, J. Garcia-Olmo, M.A. Gómez-Nieto, Analytica Chemica Acta 527, 7 (2004)
[3] J. Herrera, A. Guesalaga, E. Agosin, Measurement Science and Technology 14, 8 (2003)

[4] M. Larrain, A. Guesalaga, E. Agosin, IEEE Trans. Instrum. Meas. 57, 2 (2008)

[5] V. González-Caballero, D. Pérez-Marín, M.-I. López, M.-T. Sánchez, Sensors 11, 12 (2011)

[6] B. Kemps, L. Leon, S. Best, J. de Baerdemaeker, B. de Ketelaere, Biosystems Engineering 105, 4 (2010)

[7] R. Ferrer-Gallego, J.M. Hernández-Hierro, J.C. Rivas-Gonzalo, M.T. Escribano-Bailón, LWT - Food Science and Technology 44, 4 (2011)

[8] M. Lafontaine, Z. Bockaj, M. Freund, K.U. Vieth, C. Negara, Optical Characterization of Materials 2, 14 (2015)

[9] G. Roby, M.A. Matthews, Australian J. Grape and Wine Research 10, 74-82 (2004)

[10] M.A. Matthews, V. Nuzzo, Acta Horticulturae 754, 423-435 (2007)

[11] G. Roby, J.F. Habertson, D.O. Adams, M.A. Matthews, Australian J. Grape and Wine Research 10, 100-107 (2004)

[12] B.G. Coombe, M. Bovio, A. Schneider, J. Exp. Bot. 38, 1789-1798 (1987)

[13] H.R. Schultz, Das Deutsche Weinmagazin 18, 40-43 (2003)

[14] B.G. Coombe, Acta Horticulturae 206, 1-13 (1987)

[15] P.G. Iland, B.G. Coombe, Am. J. Enol. Vitc. 39, 71-76 (1988)

[16] H.R. Schultz, S. Kraml, U. Werwitzke, T. Zimmer, J. Schmid, Am. J. Enol. Vitc. 51, 185-190 (2000)

[17] F. Champagnol, Éléments de physiologie de la vigne et de viticulture générale (1984)

[18] J. Marais, D. van Schalkwyk, F. October, Wynober (2005)

[19] M. Lafontaine, M. Freund, Optical Characterization of Materials 1, 115-126 (2013)

[20] D. Lehmann, M. Lafontaine, B. Gruber, H.R. Schultz, Der Deutsche Weinbau 5, 24-25 (2011)

[21] B.W. Zoecklein, T.K. Wolf, J.E. March, Y. Jasinski, Am. J. Enol. Vitc. 49, 34-43 (1998)

[22] B.W. Zoecklein, T.K. Wolf, S.E. Duncan, J.E. Marcy, Y. Jasinski, Am. J. Enol. Vitc. 49, 259-265 (1998)

[23] A. Deloire, PW Journal 2-5 (2012)

[24] B.C. Duke, C.E. Butzke, Am. J. Enol. Vitc. 49, 125134 (1998)

[25] U. Werwitzke, Geisenheimer Bericht 52, 234 (2003)

\section{Danksagung}

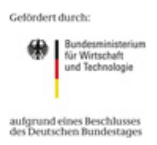

Dieses Projekt wurde gefördert durch das Bundesministerium für Wirtschaft und Technologie aufgrund eines Beschlusses des Deutschen Bundestages. 